\title{
Negative differential resistance state in the free-flux-flow regime of driven vortices in a single crystal of $2 \mathrm{H}-\mathrm{NbS}_{2}$
}

\author{
Biplab Bag, ${ }^{1, *}$ Sourav M. Karan, ${ }^{1}$ Gorky Shaw,${ }^{2}$ A. K. Sood,${ }^{3}$ A. K. Grover $\odot,{ }^{4}$ and S. S. Banerjee $\odot^{1, \dagger}$ \\ ${ }^{1}$ Department of Physics, Indian Institute of Technology, Kanpur 208016, India \\ ${ }^{2}$ National Physical Laboratory, Hampton Road, Teddington TW11 OLW, United Kingdom \\ ${ }^{3}$ Department of Physics, Indian Institute of Science, Bengaluru 560012, India \\ ${ }^{4}$ Department of Applied Sciences, Punjab Engineering College, Chandigarh 160012, India
}

(Received 9 August 2021; revised 6 October 2021; accepted 27 October 2021; published 17 November 2021)

Time series measurements [G. Shaw et al., Phys. Rev. B 85, 174517 (2012); B. Bag et al., Sci. Rep. 7, 5531 (2017)] in $2 H-\mathrm{NbS}_{2}$ crystal had unraveled a drive-induced transition wherein the critical current $\left(I_{c}\right)$ changes from a low to a high $I_{c}$ jammed vortex state, via a negative differential resistance (NDR) transition. Here, using multiple current-voltage $(I-V)$ measurement cycles, we explore the statistical nature of observing the NDR (or a quasi-NDR in reversing $I$ measurements) transition in the free-flux-flow (FF) regime in a single crystal of $2 H-\mathrm{NbS}_{2}$. Prior to the occurrence of the NDR transition, the pristine full $I-V$ curve exhibits a featureless smooth depinning from the low $I_{c}$ state. With subsequent current cycling, the NDR transition appears in the $I-V$ curve. Post-NDR, the full $I-V$ curve is seen to be noisy with depinning commencing from the higher $I_{c}$ state. The probability of observing the NDR transition always remains finite for a vortex state created with either fast or slow rate of magnetic field, $\dot{B}$. The probability of observing the NDR transition in the FF regime is found to systematically increase with magnetic field $(B)$ in weak collective pinning regime. In the strong pinning regime, the said probability becomes field independent. Retaining of a nonzero probability for the occurrence of the NDR transition under all conditions, the observed new data shows that the $I-V$ branch with higher $I_{c}$ is the more stable compared to the lower $I_{c}$ branch. We show that the higher $I_{c}$ state, generated via the NDR transition, is unique and cannot be accessed via any conventional route, in particular, by preparing the static vortex state with a different thermomagnetic history. While the $I-V$ curves do not distinguish between zero field cooled (ZFC) and field cooled (FC) modes of preparing the vortex state, the probability for observing an NDR transition has different $B$ dependences for the vortex matter prepared in the ZFC and FC modes. We find that the NDR transition occurs in a high dissipation regime, where the flow resistivity is well above the theoretical value expected in the FF regime. We understand our results on the basis of a rapid drop in vortex viscosity at high drives in $2 \mathrm{H}-\mathrm{NbS}_{2}$, which triggers a rapid increase in the vortex velocity and reorganization in the moving vortex matter leading to a dynamical unstable vortex flow. This dynamical instability leads to the NDR transition into a high entropy vortex state with high $I_{c}$.

DOI: $10.1103 /$ PhysRevB.104.184510

\section{INTRODUCTION}

In recent times, the study of transition-metal dichalcogenides (TMDs), $M X_{2}(M=\mathrm{V}, \mathrm{Nb}, \mathrm{Ta}$ and $X=\mathrm{Se}$ or $\mathrm{S})$ is experiencing a renewed interest $[1,2]$. One reason for this interest is that these materials can be easily exfoliated down to a monolayer [3-5]. Therefore, in these systems, it becomes possible to explore interesting electronic properties in two dimensions, which traditionally have been studied in bulk form of TMDs, like the Mott-insulating phase, superconductivity, and charge density waves (CDW) state [6-8]. Interestingly, out of the Nb- and Ta-based TMDs, $2 H-\mathrm{NbS}_{2}$ is the only layered superconductor in which the superconducting gap is not modulated by an underlying CDW [9]. The $2 \mathrm{H}-\mathrm{NbS}_{2}$ is a

\footnotetext{
${ }^{*}$ Present Address: Department of Condensed Matter Physics and Materials Science, Tata Institute of Fundamental Research, Mumbai 400005, India.

†satyajit@iitk.ac.in
}

two-gapped superconductor with gap energies of $0.97 \mathrm{meV}$ and $0.53 \mathrm{meV}$ [9]. Unlike $2 \mathrm{H}-\mathrm{NbSe}_{2}$ where a star-shaped vortex core structure is found due to the influence of the $\mathrm{CDW}$ [10], the high-resolution STM studies [9] in $2 \mathrm{H}-\mathrm{NbS}_{2}$ show that the vortex cores in it are circular shaped with quasiparticle bound states within a core. Recently, it has also been shown for doped $2 \mathrm{H}-\mathrm{NbSe}_{2}$ that magnetic impurities affect the nature of bound states inside the vortex core [11]. All of these suggest that it is worthwhile asking if some of the above peculiar features of $2 \mathrm{H}-\mathrm{NbS}_{2}$ affect the nature of the driven vortex state in this system. Unfortunately, only a few recent studies exist on the nature of driven vortex state in $2 \mathrm{H}-\mathrm{NbS}_{2}$.

A current-voltage $(I-V)$ measurement is equivalent to measuring the force $(F)$ versus the average vortex velocity $(u)$ behavior for the driven vortex state in type II superconductors, where $F(\propto I)$ is the Lorentz force driving the vortices and $u=V / B d$ ( $d$ is the separation between the $V$ contacts). The dynamics of driven vortices through a random pinning environment serve as a prototype for diverse hard and soft condensed matter systems [12-20]. Typically, when the ap- 
plied current density exceeds the critical depinning current density [21-24], vortices in the superconductor begin to move, thereby inducing a voltage. In the $I-V$ curve of $2 H-\mathrm{NbS}_{2}$ [25], one identifies a thermally activated flux-flow (TAFF) regime below a current, $I_{c r}$. Beyond a break in curvature (at $\left.I_{c r}\right)$ in $I-V$, the driven vortex state enters the free-flux-flow (FF) regime with a linear $I-V$ response. Studies in recent times showed that at field $B$, the $I-V$ of a vortex state in $2 H-\mathrm{NbS}_{2}$ exhibits a drive-induced transformation from a conventional low $I_{c}\left(I_{c}{ }^{l}\right)$ state to a high $I_{c}\left(I_{c}{ }^{h}\right)$ state $[26,27]$. The transformation is characterized by a sharp negative differential resistance (NDR) transition, which corresponds to negative $d V / d I$, i.e., a decrease in $V(\equiv u)$ when $I$ is increased. Note that in our paper the NDR refers to a sharp drop in $V$ while increasing $I$, and a quasi-NDR refers to a sharp drop in $V$ observed while reducing $I$ in an $I-V$ measurement. The $I_{c}{ }^{h}$ state was considered to be a drive-induced state and was identified as a jammed vortex state. Measurement of time $(t)$ series of large $V$ fluctuations [ $V(t)]$ revealed unusual features near $I_{c}{ }^{h}$, viz., (i) a critical behaviorlike divergence in time scale for which the fluctuations are sustained [26], with a divergence exponent reminiscent of random organization in a driven colloidal matter [28], and (ii) the fluctuations obey a popular Gallavotti-Cohen nonequilibrium fluctuation relation [27,29]. These studies implied that the jammed state was quite distinct from the conventionally prepared pinned static vortex matter, either in field cooled or zero field cooled.

In $2 H-\mathrm{NbS}_{2}$ crystals, $V(B)$ measurements at constant $I$ as well as $I-V$ measurements at constant $B[26,27]$ showed that the static vortex matter prepared using ZFC mode, in which $B$ was reached using a field sweep rate $(\dot{B})<0.02 \mathrm{~T} / \mathrm{min}$, depinned from a high $I_{c}$ state. However, for $\dot{B} \gg 0.02 \mathrm{~T} / \mathrm{min}$, the ZFC vortex state (for the same $B$ ) depinned from a low $I_{c}$ state. For $\dot{B} \approx 0.02 \mathrm{~T} / \mathrm{min}$, depinning commenced at $I_{c}{ }^{l}$. However, at higher drives, the moving vortex matter abruptly came to a halt and transformed into the jammed state with higher $I_{c}\left(I_{c}{ }^{h}\right)$. It may be mentioned in these earlier studies $[26,27]$, there was no detailed exploration of the nature of $I-V$ curves for the field cooled (FC) vortex matter. In these earlier studies, one only verified that by waiting long enough in the $V(t)$ measurements, the driven FC state at $0.8 \mathrm{~T}$ transforms back to a high $I_{c}$ state, which exhibits similar features in the $V(t)$ measurements as those found for the ZFC state. A detailed investigation of the conditions under which the unusual NDR transition occurs and its dependence on $B, \dot{B}$ and the thermomagnetic history of the starting static vortex state, has been lacking.

It is pertinent to recall here a few general features known from studies on the vortex state. In realistic samples with moderate to strong pinning, the $I_{c}$ of the static ZFC vortex state is usually lower [30-32] than that for the FC state [30-32], as during field cooling, the pinning centers freeze in a more spatially disordered pinned vortex configuration leading to a higher $I_{c}$. Only in very clean samples, the $I_{c}$ of the ZFC and FC states are undiscernibly close. Usually, a high $\dot{B}$ results in annealing of disordered vortex state into an ordered state with low $I_{c}$ [30-33]. The glassy vortex state exhibits memory and metastability effects [31,32]. Quite a few of these features have been explained via an edge contamination process [34,35], an ever-present process in the driven (with transport current) vortex state. In $I-V$ measurement, there is a continuous injection of disordered phase from irregular sample boundaries into the vortex state in the sample at a field $B$ and temperature $T$. Within this process, a low $I_{c}$ is favored, when the rate at which the disordered phase is injected from the irregular sample edges is slower than the rate at which the injected disordered phase anneals, as it mixes with an ordered vortex phase already present inside the sample. If the order of the above rates reverses, then a disordered or an admixed vortex phase is favored. The annealing rate also depends on the $(B, T)$ of the static vortex state, as the annealing rate is faster for an elastic vortex solid, while it is slower for a softened vortex state. In the backdrop of these features, it may be worthwhile to further investigate the $I-V$ features in $2 \mathrm{H}-\mathrm{NbS}_{2}$.

Using high sensitive dc magneto-transport measurements in a $2 \mathrm{H}-\mathrm{NbS}_{2}$ single crystal, we explore the statistics of observing the NDR transition in the FF regime and study its dependence on $B, \dot{B}$, and the thermomagnetic history of the static vortex state. We find that the $I-V$ curves are identical for the FC and ZFC vortex states. We examine features developing in the $I-V$ with repeated current cycling between zero and high current value (without causing heating at the contacts). Our study shows that the NDR (or a quasi-NDR) transition may not appear in the first $I-V$ run, and a few $I$ cycles are required to generate the NDR transition in the FF regime. These studies determine the $B$ dependence on the number of the current cycles $(N)$ needed to generate an NDR (or a quasi-NDR) transition. The $N$ is inversely related to the probability of observing an NDR (or quasi-NDR) transition. We find statistically that this probability decreases with an increase in $B$. We also find that irrespective of the value of $\dot{B}$ value, with a sufficient number of $I$ cyclings, there is always a nonzero probability for observing an NDR (or quasi-NDR) transformation. The above results cannot be fully reconciled within the edge contamination process alone. While the $I-V$ curves exhibit identical features for the vortex matter prepared via ZFC and FC modes, the $N(B)$ shows distinct behavior for the ZFC and FC curves. The difference in ZFC-FC behavior of $N(B)$ is related with a crossover from strong to weak pinning regime of the underlying static vortex solid. We discuss our results in terms of dynamical instability in the vortex flow present in $2 \mathrm{H}-\mathrm{NbS}_{2}$. The dynamical instability triggers the NDR transition into an unusually high entropy vortex configuration with a high $I_{c}$.

\section{EXPERIMENTAL DETAILS}

We perform four-probe transport measurements in a single crystal of $2 H-\mathrm{NbS}_{2}$ superconductor (approximate dimension of $1.9 \times 1.0 \times 0.045 \mathrm{~mm}^{3}$ ) by passing current in the basal $(a b)$ plane of the single crystal with $B$ applied along the crystallographic $c$ axis $(B \| c)$. In Sec. I of the Supplemental Material [36], we also show results on another crystal (A2) from different batch with dimensions $0.9 \times 0.9 \times$ $0.045 \mathrm{~mm}^{3}$. The single crystals were grown using the chemical vapor transport technique with growth details reported elsewhere [37]. The powder XRD analysis of $2 \mathrm{H}-\mathrm{NbS}_{2}$ samples from different batches as well as the energy dispersive X-ray analysis (EDX) study are presented in Sec. II of the 
Supplemental Material [36]. The study shows the presence of only the $2 \mathrm{H}-\mathrm{NbS}_{2}$ superconducting phase. The zero-field superconducting transition temperature $\left(T_{c}\right)$ and the residual resistivity ratio $[\mathrm{RRR} \equiv R(300 \mathrm{~K}) / R(10 \mathrm{~K})]$ of the present sample are $5.8 \pm 0.1 \mathrm{~K}$ and 25 , respectively (in crystal A2, $T_{c}$ and RRR are $5.8 \mathrm{~K}$ and 35). Four-probe electrical contacts are made on a freshly cleaved sample surface using low-temperature silver epoxy with a mean spacing between the voltage contacts, $d=0.55 \pm 0.01 \mathrm{~mm}$ (contact resistance $\sim 10 \pm 4 \mathrm{~m} \Omega$ ). The $I_{c}$ is determined from $I-V$ measurements using a voltage criterion, viz., $V \geqslant 2 \mu \mathrm{V}$ at $I=I_{c}$. Before the $I-V$ measurements, the pristine static vortex matter is prepared via either ZFC or FC mode at a field $B$. The $I-V$ of different ZFC vortex states, prepared by reaching the target field $B$ using different field sweep rates $\dot{B}$, are studied. In our paper we have studied different field sweep rates. A field sweep rate of $0.1 \mathrm{~T} / \mathrm{min}$, corresponds to induced emf of the order of $3.2 \mathrm{nV}$. A current of $10 \mathrm{~mA}$ (for the crystal whose results are shown in the paper) corresponds to a current density of $2.2 \times 10^{5}$ A.m $^{-2}$ (note that due to the nearly comparable cross section of both the crystals whose data are shown in the paper and in Supplemental Material [36], the current densities for both crystals are nearly similar). However, we would like to mention here that we do not record any $I-V$ measurements while the field is being swept towards the target field. After reaching the target field value, the field is stabilized with a wait time of up to $5 \mathrm{~min}$. After this wait time, we perform the $I-V$ measurement. Hence, the emf of $3.2 \mathrm{nV}$ induced by field sweeping is a transient, which does not affect our $I-V$ measurements. Furthermore, these transient voltages are far smaller than the voltages we measure.

The $I-V$ is recorded while driving the vortices by ramping up (or down) the dc applied current. For all $I-V$ measurements, the $I$ sweep rate was $\sim 10 \mathrm{~mA} / \mathrm{min}$. The $I-V$ measurement for the forward (increasing $I$ ) and reverse (decreasing $I$ ) runs labeled as F\# and R\#, where "\#" is a number referring to the leg of the current cycle (see subsequent text). The present crystal is from the same batch as the A1 crystal on which time-series measurements related to the jamming transition had been reported in Ref. [27].

\section{RESULTS}

\section{A. Generating the NDR transition in $I-V$ and the field dependence of high critical current state}

Our earlier measurements [26] on another crystal of $2 H-\mathrm{NbS}_{2}$ had explored the jamming transition, which was an abrupt jump to a low $V$ state, as seen in an isothermal $V$ - $B$ measurement. In Ref. [26], using $I-V$ measurements it had shown that the transition was associated with a field sweep rate-dependent transformation into a high $I_{c}$ state. The work, however, focused on characterizing the nature of the nonequilibrium jamming transition via detailed time-series voltage measurements $[26,27]$. The instabilities in $I-V$ characteristics due to jamming are explored in greater detail in this paper. Figure 1(a) shows the $I-V$ response in log-linear scale for a ZFC vortex state prepared at $T=2.5 \mathrm{~K}$ and $0.3 \mathrm{~T}$ with $\dot{B}=0.03 \mathrm{~T} / \mathrm{min}$. It may be recalled that earlier studies in the sample A1 suggested that in $I-V$ for $\dot{B}>0.02 \mathrm{~T} / \mathrm{min}$, no

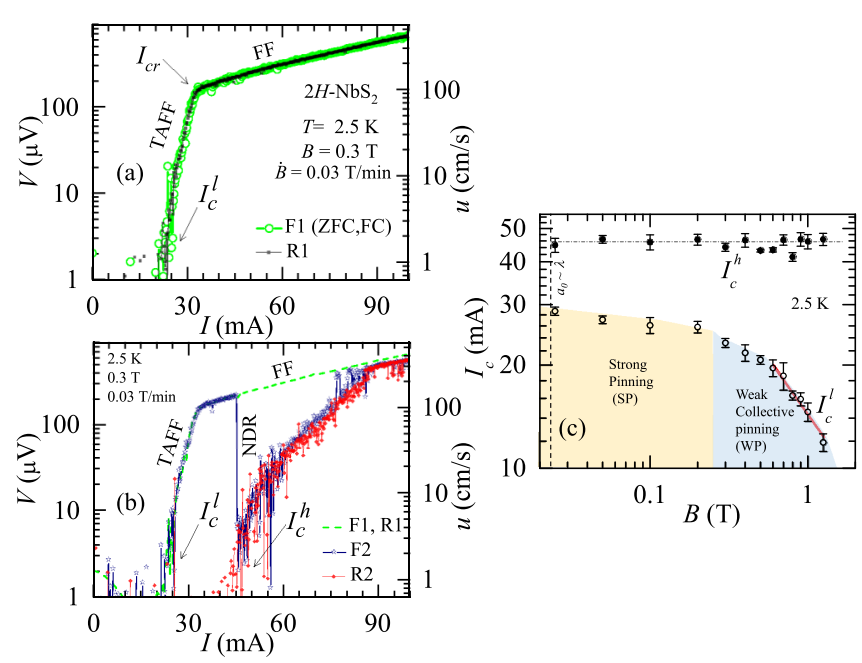

FIG. 1. The left and right $y$ axes show the voltage $V$ and the corresponding vortex velocity $u$ data versus $I$ (plotted in a log-linear scale) for the driven vortex state at $2.5 \mathrm{~K}$ and $0.3 \mathrm{~T}$ for (a) F1 and R1 (see text for details), and (b) F1, R1, F2, and R2 runs. The pristine vortex state at $B=0.3 \mathrm{~T}$ is reached with a $\dot{B}=0.03 \mathrm{~T} / \mathrm{min}$. In (a), the F1 curve represents the $I-V$ response for ZFC and FC pristine static vortex matter. In (b), the green dashed line represents the F1 runs of (a). The locations of $I_{c}^{l}, I_{c r}$ and $I_{c}^{h}$ are marked with arrows in (a) and (b). (b) shows the NDR transition into the high $I_{c}$ state in the F2 and R2 runs. (c) shows the $B$ dependence of $I_{c}^{l}$ and $I_{c}^{h}$ estimated from isothermal $I-V$ 's at $2.5 \mathrm{~K}$. The thick red line presents $I_{c}^{l} \propto B^{-\alpha}$ behavior with $\alpha=0.6 \pm 0.1$. The yellow and blue regions identify strong pinning (SP) regime and weak collective pinning (WP) regime, respectively. The vertical dashed line represents the $a_{0} \sim \lambda_{a b}$ line.

NDR transition into the high $I_{c}$ state was seen (see Fig. 2 of Ref. [26]). In our subsequent measurements in the present sample here, we label the first forward $I-V$ measurement as the F1 run, where $I$ is being increased from zero up to 100 $\mathrm{mA}$. We would like to mention that in the F1 measurements, we observe that whether we prepare the static vortex state in ZFC or FC mode, the measured depinning threshold of the pristine static state $\left(I_{c}^{l}\right)$ for both these modes are identical, and the corresponding $I-V$ curves overlap. We identify this $I-V$ curve as the low $I_{c}$ branch of $I-V$. The $y$ axis on the right-hand side of Fig. 1(a) presents the corresponding $u(I)$ values determined from the $I-V$. As $I$ is increased from zero in the F1 run, the vortex state depins at $I_{c}^{l} \sim 23 \mathrm{~mA}$. After depinning, the moving vortex matter exhibits a linear TAFF regime. The linear behavior in the log-linear scale suggests an Arrhenius-like thermally activated motion [25] until the knee in the $I-V$ is reached, viz., at $I_{c r} \sim 34 \mathrm{~mA}$.

In Fig. 1(a), the $I-V$ characteristic for $\mathrm{F} 1$ has a break in slope at $I_{c r}$, which corresponds to a crossover from TAFF into the free-flux-flow (FF) regime [25]. We observe a monotonic increase in $u$ with drive in this FF regime as $I$ is ramped up to $100 \mathrm{~mA}$. Typically, the FF regime is considered a uniform vortex flow regime. The low $I_{c} I-V$ branch, in this FF regime, is completely reversible while increasing (F1) and decreasing (R1) the current between $I_{c r}$ and $100 \mathrm{~mA}$. During R1 $(I: 100 \mathrm{~mA} \rightarrow 0 \mathrm{~mA}$ ), the $I-V$ curve completely overlaps 
with the forward one (F1) run [see Fig. 1(a)]. Note, the $V(I)$ falls below the noise level $(<2 \mu \mathrm{V})$ at $I_{c}{ }^{l} \sim 23 \mathrm{~mA}$ for R1 run as well. The complete overlap between the F1 and R1 curves in Fig. 1(a) rules out any significant Joule heating effect at contacts.

In Fig. 1(b), the second $I-V$ cycle F2 (viz., $I$ is again increased from zero towards $100 \mathrm{~mA}$ ) exhibits features that are similar to $\mathrm{F} 1$ and the vortex state depins at $I_{c}{ }^{l} \sim 23 \mathrm{~mA}$. However, for the F2 run above $I_{c r}$, as the driven vortex state enters the FF regime [Fig. 1(b)], $I-V$ exhibits an abrupt NDR transition at $I \sim 45 \mathrm{~mA}$ and the average vortex velocity abruptly drops down from $100 \mathrm{~cm} / \mathrm{s}$ (in the FF regime) to 4 $\mathrm{cm} / \mathrm{s}$. With the subsequent increase of $I$ beyond $45 \mathrm{~mA}$, the $V(I)$ increases gradually, however, there is a lot more voltage noise compared to the smooth $I-V$ while depinning at $I_{c}^{l}$. The large $V(I)$ or $u(I)$ fluctuations imply instability in vortex flow beyond the NDR transition at $45 \mathrm{~mA}$. It appears that between $\sim 45 \mathrm{~mA}$ and $\sim 80 \mathrm{~mA}$, the $I-V$ curve along the FF branch in the $\mathrm{F} 1$ run is unstable. Beyond $80 \mathrm{~mA}$, the fluctuating $I-V$ in F2 merges with that of the FF regime [Fig. 1(b)]. Upon reducing current down from $100 \mathrm{~mA}$ (R2 run), $I-V$ response displays similar $V$ fluctuations as in the F2 run. Also, the $V(I)$ for the R2 run falls below the noise level $(<2 \mu \mathrm{V})$ at $I \leqslant 44 \mathrm{~mA}\left(\equiv I_{c}{ }^{h}\right)$, indicating driven vortices enter the high $I_{c}$ state. It is important to note that after the onset of this high $I_{c}$ vortex state in R2 run, the driven vortex matter in subsequent $I$ cycles $(0 \rightarrow 100 \rightarrow 0 \mathrm{~mA} \rightarrow \ldots)$ depins only from the high $I_{c}$ state, and depinning from the low $I_{c}$ state (at $I_{c}{ }^{l}$, observed for the pristine F1 run) is never observed again. We call this the high $I_{c}$ branch of the $I-V$ curve. Thus, with sufficient number of current cycling, the NDR is always encountered in the FF regime with $\dot{B}=0.03 \mathrm{~T} / \mathrm{min}$, and the $I-V$ curve displays irreversible behavior with two branches of the $I-V$ curve (one being the low $I_{c}$ and the other is the high $I_{c}$ branch of the $I-V$ curve).

In Fig. 1(c), we find that with increasing $B, I_{c}^{l}(B)$ varies as $1 / B^{\alpha}$, where $\alpha=0.6 \pm 0.1$ for the red line in Fig. 1(c). This form of the $I_{c}{ }^{l}(B)$ represents the weak collective pinning regime of the elastic vortex matter $[21,22,24]$. Within the region shaded light blue in Fig. 1(c), $I_{c}^{l}(B) \propto 1 / B^{\alpha}$. In Fig. 1(c), we show the dashed vertical as the field values where the intervortex spacing, $a_{0} \sim \sqrt{\frac{\phi_{0}}{B}}=\lambda_{a b}$ (where $\phi_{0}$ is the magnetic flux quantum and $\lambda_{a b}$ is $a b$ plane penetration depth), and using $\lambda_{a b} \sim 350 \mathrm{~nm}$ in $2 H-\mathrm{NbS}_{2}$ [38], we get a $B \sim 0.016 \mathrm{~T}$. At low fields, viz., inside the light yellow regime in Fig. 1(c) (which is near $a_{0} \sim \lambda_{a b}$ line), due to relatively weak intervortex interaction, the vortex state is in a strong pinning regime. In the strong pinning regime, the pinning force almost saturates, and hence $I_{c}{ }^{l}$ has a weak field dependence in this regime. In Fig. 1(c), we note that the high $I_{c}$ state has almost no $B$ dependence and the $I_{c}{ }^{h}$ value is higher than the maximum strong pinning regime value. We would like to mention that presence of any strong pinning centers such as structural imperfections in the crystal or compositional variations will affect $I_{c}{ }^{l}$. Had these strong pinning centers played a role in reaching the high $I_{c}$ state, then we should have observed this high $I_{c}$ upon field cooling the sample. In the Sec. III of Supplemental Material [36], we show that by field cooling we do not obtain the high $I_{c}$ state, rather we get the low $I_{c}$ state.

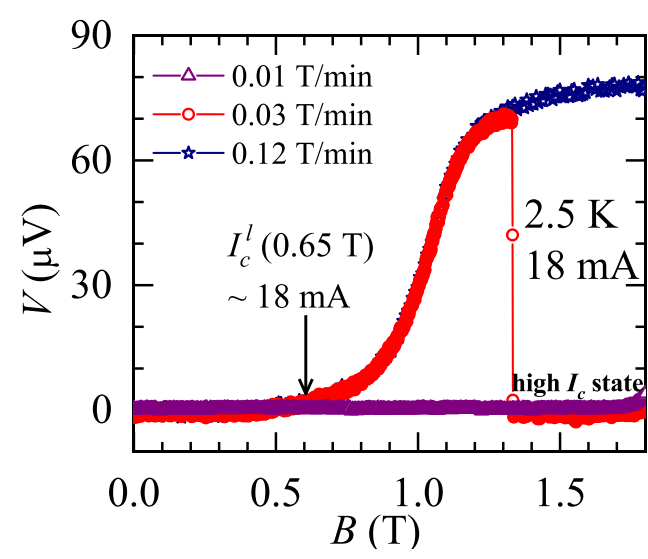

FIG. 2. $V(B)$ data at $2.5 \mathrm{~K}$ and a constant $I=18 \mathrm{~mA}$ for $\dot{B}=$ $0.01,0.03$, and $0.12 \mathrm{~T} / \mathrm{min}$. For data taken with high field sweep rate of $0.1 \mathrm{~T} / \mathrm{min}$, the $V(B)$ shows depinning $[V(B)>2 \mu \mathrm{V}]$ at a $B$ value when the $I_{c}(B)$ is less than $18 \mathrm{~mA}$. For field sweep rate of 0.03 $\mathrm{T} / \mathrm{min}$, while the vortex state depins from a low $B$, it subsequently transforms into a high $I_{c}$ state at higher $B$. With a slower field sweep rate of $0.01 \mathrm{~T} / \mathrm{min}$, the vortex state never depins as it remains in the high $I_{c}$ state (confirmed with $I-V$ measurements).

\section{B. Effect of magnetic field ramp rate on the generation of high $I_{c}$ state}

Figure 2 displays $V$ versus $B$ behavior measured with a constant $I=18 \mathrm{~mA}$ at $2.5 \mathrm{~K}$ for different $\dot{B}$ values. The observed jump in $V(B)$ and the $\dot{B}$ dependence in $V(B)$ measurement in this crystal of $2 H-\mathrm{NbS}_{2}$ is similar to the $V(B)$ behavior associated with the jamming transition shown in Fig. 1 of Ref. [26], in a different crystal of $2 \mathrm{H}-\mathrm{NbS}_{2}$. Figure 2 suggests that the jamming transition feature seen in $V(B)$ measurement corresponds with the NDR transition found in $I-V$ measurements. From Fig. 2 we see that for a ZFC vortex state prepared using a high $\dot{B}=0.12 \mathrm{~T} / \mathrm{min}$, the vortex state depins from a low $I_{c}$ value, viz., the $B$ at which $I_{c}^{l}(B)<18 \mathrm{~mA}$, depinning occurs. However, for vortex states prepared up to $1.8 \mathrm{~T}$ with low $\dot{B}=0.01 \mathrm{~T} / \mathrm{min}$, the vortex matter does not get depinned in response to drive with $I=18 \mathrm{~mA}$, thereby implying that the underlying vortex matter does not have a low $I_{c}$. This feature is consistent with the results of Ref. [26], which showed that for $\dot{B}<0.02 \mathrm{~T} / \mathrm{min}$, the vortex matter attains the high $I_{c}$ state (see inset of Fig. 1 in Ref. [26]). From the $V(B)$ response for $\dot{B}=0.01 \mathrm{~T} / \mathrm{min}$ in Fig. 2, it is clear that the vortex state remains pinned with the $V$ values staying in the noise floor, indicating the vortex state has been prepared in a high $I_{c}$ state (i.e., $I_{c}>18 \mathrm{~mA}$ for $B<1.8 \mathrm{~T}$ at $2.5 \mathrm{~K}$ ). However, at an intermediate sweep rate of 0.03 $\mathrm{T} / \mathrm{min}$, the vortex matter gets depinned from a low $I_{c}$ value and subsequently it exhibits an NDR transition at $B \sim 1.32 \mathrm{~T}$, where the voltage falls sharply below the noise level. Here, the vortex state makes a transition into the high $I_{c}$ state. Following the NDR drop at $B>1.35 \mathrm{~T}$ with $\dot{B}=0.03 \mathrm{~T} / \mathrm{min}$, a measurement of $I-V$ shows vortex state depinning at $I_{c}{ }^{h} \sim 45 \mathrm{~mA}$ (see Supplemental Material, Sec. IV [36] for the $I-V$ data). In other words, with $\dot{B}=0.03 \mathrm{~T} / \mathrm{min}$ followed by depinning at $I_{c}^{l}(B)$, at higher $B$ there is an NDR transition in $V(B)$, where the vortex matter re-organizes into the high $I_{c}$ state. 

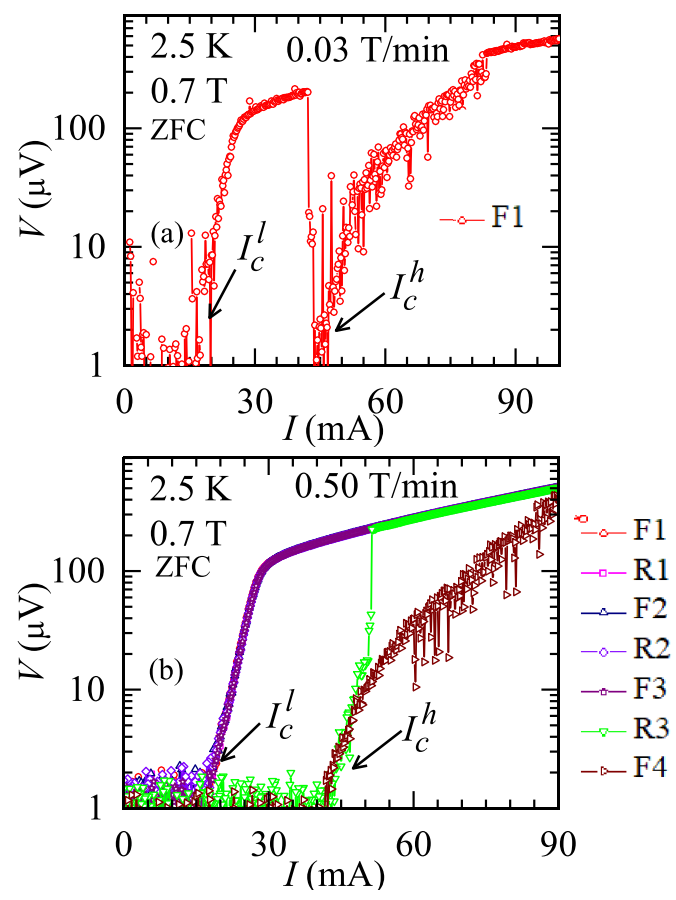

FIG. 3. I-V data (for F1, R1, F2, R2, F3, R3, and F4 runs) at $2.5 \mathrm{~K}$ and $0.7 \mathrm{~T}$ (ZFC) for (a) $\dot{B}=0.03 \mathrm{~T} / \mathrm{min}$ and (b) $0.50 \mathrm{~T} / \mathrm{min}$, respectively. The locations of $I_{c}^{l}$ and $I_{c}^{h}$ are marked with arrows. (see text for details).

Figures 3(a), 3(b) show the $I-V$ data for a ZFC static vortex state prepared at $2.5 \mathrm{~K}$ and $0.7 \mathrm{~T}$ with $\dot{B}=0.03 \mathrm{~T} / \mathrm{min}$ and $0.5 \mathrm{~T} / \mathrm{min}$, respectively. Figure 3(a) shows that for the F1 run after depinning from a low $I_{c}{ }^{l} \sim 17 \mathrm{~mA}$, the $I-V$ exhibits an NDR transition at $41 \mathrm{~mA}$ (from the FF regime), and the driven vortex matter enters the high $I_{c}$ state $\left(I_{c}{ }^{h} \sim 45 \mathrm{~mA}\right)$. By comparing with Fig. $1(B=0.3 \mathrm{~T})$, it appears that for the same $\dot{B}$, a lesser number of current cycles is required to observe the NDR transition at higher $B$ values, viz., the probability for observing the NDR transition in the $I-V$ increases with $B$. When the same $B=0.7 \mathrm{~T}$ is reached with a very high $\dot{B}=0.5 \mathrm{~T} / \mathrm{min}$, which is almost 25 times the value of 0.02 $\mathrm{T} / \mathrm{min}$ of Ref. [26] below which there is a transformation from the low to high $I_{c}$ state, one had not expected to observe any NDR transition into a high $I_{c}$ state. However, the vortex state prepared at $2.5 \mathrm{~K}$ and $0.7 \mathrm{~T}$ with high $\dot{B}=0.5 \mathrm{~T} / \mathrm{min}$, in Fig. 3(b) shows a depinning from the low $I_{c}{ }^{l}$ state. The $I-V$ curves remain reversible over multiple $I$ cycles initially. This reversible feature is survived only until the third forward run (F3). In the third reverse run (R3), the high $I_{c}$ state resurfaces via the quasi-NDR feature. After this quasi-NDR transition, upon reducing the $I$ to zero and in the subsequent F4 run, the depinning occurs at $I_{c}{ }^{h} \sim 45 \mathrm{~mA}$. It is clear that with a sufficient number of $I$ cyclings, the higher $I_{c}$ branch of the $I-V$ curve will be reached via an NDR (or quasi-NDR) transition.

\section{Variations of depinning characteristics across $I_{c}{ }_{c}$ and $I_{c}{ }^{h}$ with $B$ and the $N(B)$ plot}

Figures 4(a)-4(d) show the $I-V$ of the driven vortex state at $T=2.5 \mathrm{~K}$ for $B=0.1,0.4,0.8$, and $1.25 \mathrm{~T}$ (ZFC state with ramp rate $\dot{B}=0.03 \mathrm{~T} / \mathrm{min}$ ), respectively. At $B=0.1 \mathrm{~T}$,

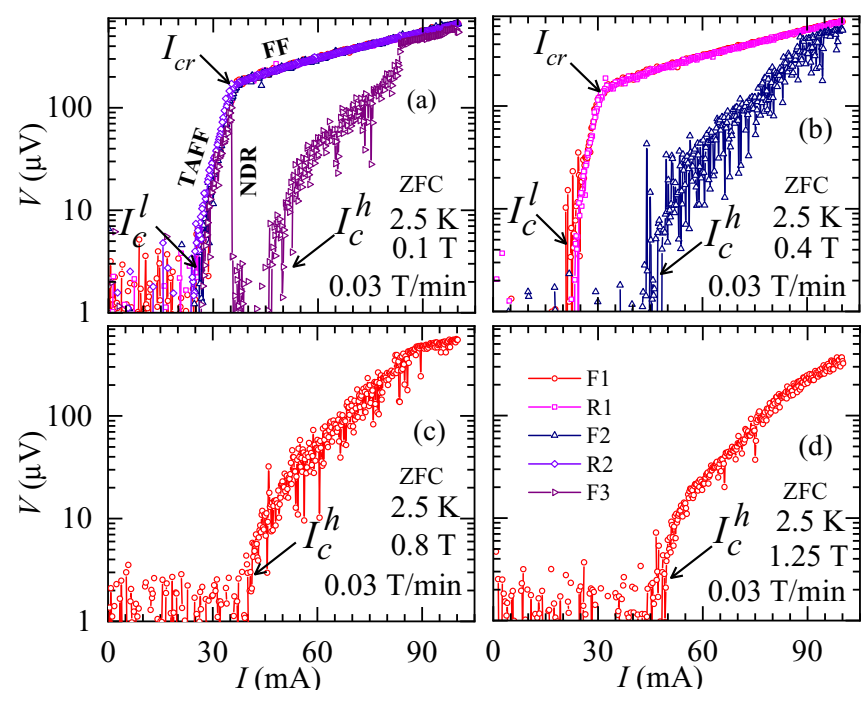

FIG. 4. $I-V$ data of the driven vortex matter at $2.5 \mathrm{~K}$ and (a) 0.01 $\mathrm{T}$, (b) $0.04 \mathrm{~T}$, (c) $0.8 \mathrm{~T}$, and (d) $1.25 \mathrm{~T}$. The pristine vortex state is prepared in ZFC mode at $\dot{B}=0.03 \mathrm{~T} / \mathrm{min}$. $I_{c}^{l}$ and $I_{c}^{h}$ locations are marked with arrows. The current cycle legends shown in (d) are retained the same for (a)-(d).

Fig. 4(a) shows that the $I-V$ characteristics overlap and exhibit identical features for the initial four runs $(F 1, R 1, F 2$, and $\mathrm{R} 2)$. However, in the F3 run, the driven vortices exhibit NDR transition, and the vortex matter enters the high $I_{c}$ state with $I_{c}{ }^{h}=46 \mathrm{~mA}$, followed by large fluctuations in voltage. The time series of these $V$ (or $u$ ) fluctuations around the jammed state near $I_{c}{ }^{h}$ (data not being shown here) were similar to those reported earlier [26,27]. For all subsequent runs, we observe the vortex state depinning at $I_{c}{ }^{h}$. Figure 4(b) shows that at $0.4 \mathrm{~T}$, the vortex state depins from low $I_{c}$ state in F1and R1 runs and the high $I_{c}$ state is attained on F2 run. Figures 4(c), 4(d) show that the pristine vortex matter gets depinned at $I_{c}{ }^{h}=46.5 \mathrm{~mA}$, and we did not observe depinning from a low $I_{c}$ value at these relatively higher magnetic fields of 0.8 and $1.25 \mathrm{~T}$.

Based on all $I-V$ data at various $B$, we count the number of $I$ cycles required to reach $I_{c}{ }^{h}$ state via NDR-like transition as a function of $B$ (at $2.5 \mathrm{~K}$ ). We denote these cycles as $N$ for each complete $I$ - $V$, viz., $N=1$ when the $I_{c}{ }^{h}$ state is reached via the NDR transition in the first forward $I-V$ run, $\mathrm{F} 1$, or $N=2$ when the $I_{c}{ }^{h}$ state is reached via the quasi-NDR transition in the first reverse $I-V$ run, R1 and so on. The $N$ count is inversely proportional to the probability of observing the NDR (quasi-NDR) transition in the FF state. We treat NDR or quasi-NDR on the same footing as they are associated with the same phenomenon. The main panel of Fig. 5 shows the behavior of $N(B)$ for the ZFC state with $\dot{B}=0.03 \mathrm{~T} / \mathrm{min}$ at $2.5 \mathrm{~K}$. The ZFC state in Fig. 5 shows $N(B)$ decreases with increasing $B$ and $N(B)=1$ for $B \geqslant 0.6 \mathrm{~T}$. This shows that in $2 \mathrm{H}-\mathrm{NbS}_{2}$, the FF state at higher fields $(>0.6 \mathrm{~T})$ is more susceptible to exhibit the NDR transition to produce the high $I_{c}$ state compared to that at lower $B$ for the same field sweep rate of $0.03 \mathrm{~T} / \mathrm{min}$. The inset panel of Fig. 5 shows that over the whole $B$ range (from $0.025 \mathrm{~T}$ to $1.25 \mathrm{~T}$ ), $N(B)$ is almost uniform (at four cycles) for the FC vortex state, while the 


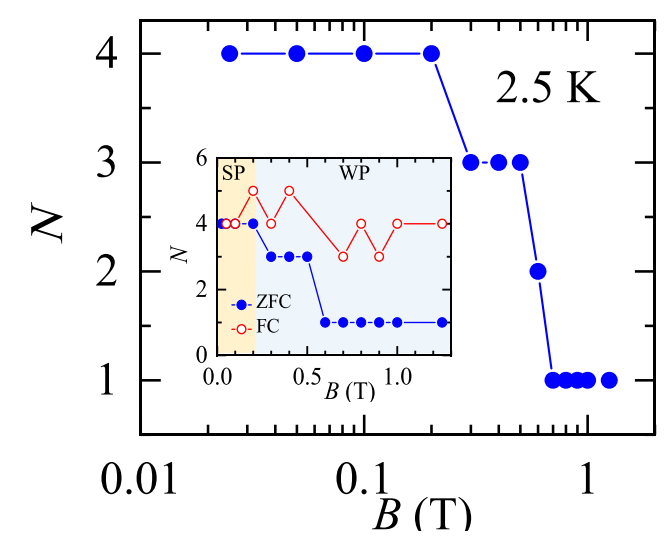

FIG. 5. Main panel shows the number of $I$ cycles needed for the $I-V$ to show the NDR transition, i.e., $N(B)$ at $2.5 \mathrm{~K}$ (for the static vortex state prepared in the ZFC mode). Inset shows comparison of $N(B)$ values for static vortex state prepared in the FC and ZFC modes at $2.5 \mathrm{~K}$. In the inset, the yellow and blue regions represent the SP and WP regimes [compared with Fig. 1(c)], respectively.

$N(B)$ decreases with $B$ (above $0.2 \mathrm{~T}$ ) for the ZFC state. Thus, although the nature of $I-V$ curves is similar for the ZFC and FC states, the probability of observing the NDR transition is different for these two thermomagnetic history states. For our measurements, we also know that statistically, at any $B$, the probability of observing an NDR transition always has a nonzero finite value at any $\dot{B}$. A vortex state produced at a fixed $B$, which is reached with a high $\dot{B}$, the probability for observing the NDR transition in its $I-V$ is low [i.e., the $N(B)$ value is high], while the probability is high for a smaller $\dot{B}$. The finiteness of the probability for observing the NDR transition confirms that the high $I_{c}$ branch of the $I-V$ curve is a more stable one compared to the low $I_{c}$ branch. We would like to mention here that between samples taken from three different batches of $2 H-\mathrm{NbS}_{2}$ crystal, the difference in $I_{c}{ }^{l}$ values between the different crystals is within about $5 \mathrm{~mA}$ [27]. In our paper by varying $B$, in the same crystal we are able to study the behavior of $N(B)$ alongside wide variation in $I_{c}^{l}$, which also encompass different pinning regimes. In Sec. I of the Supplemental Material [36], we show the transformation into the high $I_{c}$ state from the low $I_{c}$ state by repeated $I-V$ cycling for a sample (A2) taken from a different sample batch. In Sec. V of the Supplemental Material [36], we show all I-V's corresponding to the data shown in Fig. 5. In Sec. VI of the Supplemental Material [36], we show that once the high $I_{c}$ state is reached after four $I-V$ cycles, this state is retained even with additional $I-V$ cyclings (five more $I-V$ cyclings). In Sec. VII of the Supplemental Material [36], we show that over the entire $I-V$ measurement, the fluctuations in sample temperature are well below $5 \mathrm{mK}$.

\section{DISCUSSION}

Our Fig. 1(c) shows that most of the $B$ values, at which we perform our experiments, are well in the elastic vortex regime. As per the edge contamination model, for an elastic vortex state (ordered), a low $I_{c}$ state is reached if the disordered phase is injected into the sample from the edges at a rate (controlled by $\dot{B}$ ) slower than the annealing rate of the injected disorder in the elastic vortex state $[34,35]$. We have already shown in Fig. 3 that irrespective of the field sweeping rate, the driven vortex matter always transforms into a high $I_{c}$ state with repeated cyclings of current. From the edge contamination process [34,35], a fast field sweep rate triggers an annealing transformation of the injected disordered state into an ordered state in the elastic regime of the vortex state, viz., fast field sweep rates favor a high to low $I_{c}$ transformation [30,31]. However, here in $2 \mathrm{H}-\mathrm{NbS}_{2}$, we see the opposite feature, viz., even with very fast field sweep rates there is a transformation from the low to high $I_{c}$ state. In fact, the high $I_{c}$ state is the more stable branch of the $I-V$ curve. All of these show that, although it is an ever-present process in all $I-V$ measurements, our results are not fully explained within the edge contamination process [34,35].

In the FF regime, the flux-flow resistivity $\left(\rho_{f}\right)$ is related to normal state resistivity $\left(\rho_{n}\right)$ via, $\rho_{f}=\rho_{n} \frac{B}{B_{c 2}}$ where $B_{c 2}$ is the upper critical field [39]. Studies in high $T_{c}$ superconductors have shown that at low temperatures $\left(T \ll T_{c}\right)$, using large pulsed currents, it is possible to attain flow resistivity, $\rho \sim 2 \rho_{f}$ in FF regime. Such studies in HTSC and MoGe thin films [40-43] showed that at $\rho / \rho_{f} \sim 2$ an NDR transition occurred. These studies conclude that a reduction of the viscosity of the moving vortices leads to a rapid rise in vortex velocity. At large flow velocities, dynamic instabilities in driven vortex state lead to an NDR event [40-47]. However, it must be mentioned here that unlike the NDR transition in $2 \mathrm{H}-\mathrm{NbS}_{2}$, no high $I_{c}{ }^{h}$-like state was reported to have been reached via the NDR transition in HTSC. In $2 H-\mathrm{NbS}_{2}$, with $\rho_{f}=60 \mu \Omega-\mathrm{cm}$, one finds that near the onset of NDR transition in the FF regime, $\rho / \rho_{f} \sim 7$ [see Fig. S6, Sec. VIII of Supplemental Material [36]]. In $2 \mathrm{H}-\mathrm{NbS}_{2}$, the high $\rho / \rho_{f}$ value is reached quite easily with comparatively much lower currents than in HTSCs [40-43]. We present here a possible scenario to explain the high $\rho / \rho_{f}$ values reached in $2 \mathrm{H}-\mathrm{NbS}_{2}$. Local density of states studies using STM have shown localized bound states within the vortex core in $2 \mathrm{H}-\mathrm{NbS}_{2}$ [9]. The absence of any underlying CDW in $2 \mathrm{H}-\mathrm{NbS}_{2}$ (which modifies the spectrum of these quasiparticle bound states) perhaps favors more weakly localized quasi-particles within its vortex cores compared to other materials.

In the driven vortex state, Larkin-Ovchinnikov (LO) [48] proposed that the electric field acting across the vortex cores accelerates the quasiparticles in them. Once these bound quasiparticles acquire sufficient energy, they delocalize out of the vortex cores. In $2 \mathrm{H}-\mathrm{NbS}_{2}$, due to the weakly bound nature of the quasiparticles in the vortex core, low drives may be sufficient to delocalize the quasiparticles. Due to this delocalization, LO [48] considered a nonthermal quasiparticle distribution around the vortex, which causes the vortex core to shrink, and hence a significant drop in the vortex viscosity. The conditions for attaining the nonthermal quasiparticle distribution is that the electron-electron scattering time, $\tau_{e e}$ is greater than the electron-phonon scattering time, $\tau_{e p}$. Using the expression for $\tau_{e e}$ and $\tau_{e p}$ in Refs. [42,49], we estimate for $2 H-\mathrm{NbS}_{2}, \tau_{e e} / \tau_{e p}=\frac{1}{r^{2}} \frac{E_{F} T}{k_{B} T_{D}^{2}}=\frac{7.73}{r^{2}}>1$, where, the Fermi energy $E_{F}=30 \mathrm{eV}$ [50], Debye temperature $T_{D}=300 \mathrm{~K}$ [51], $T=2 \mathrm{~K}$ and $r<1$ is a dimensionless parameter related to 
phonon reflection coefficient at boundaries [42]. It is clear that for $2 \mathrm{H}-\mathrm{NbS}_{2}$, the value of $\tau_{e e} \gg \tau_{e p}$ favors a shrink in vortex core, which decreases the vortex viscosity, and hence significantly speeds up the vortices. The increased speed of the vortices results in enhanced flow resistivity. Recall from Fig. 1(b) in $2 H-\mathrm{NbS}_{2}, u=100 \mathrm{~cm} / \mathrm{s}$ near NDR, compared to $u \sim 1 \mathrm{~cm} / \mathrm{s}$ in $2 H-\mathrm{NbSe}_{2}$ [52]. The rapid (fast $u$ ) injection of vortices from the irregular sample edges would trigger a rapid reorganization of vortices within the moving vortex state in the sample. The lowered viscosity results in unstable flow and the NDR transition [40-47] in $2 \mathrm{H}-\mathrm{NbS}_{2}$. The reorganization timescale is of the order of, $\tau_{\text {re-org }} \sim\langle\delta\rangle / u$, where $\langle\delta\rangle$ is the mean spacing of vortices in the sample. As $\langle\delta\rangle \sim a_{0} \propto 1 / \sqrt{B}$, hence $\tau_{\text {re-org }} \propto 1 / \sqrt{B}$. A decrease in $\tau_{\text {re-org }}$, at higher $B$ (for fixed $u$ ) or high $u$ (at fixed $B$ ), corresponds to a higher probability ( $\propto 1 / \tau_{\text {re-org }}$ ) for triggering rapid vortex reorganization in the flowing vortex state. This emerging dynamic instability triggers the NDR transition event (recall $N$ is inversely proportional to the probability of observing NDR).

For a static elastic vortex matter in a random pinning environment, the ZFC and FC vortex states have different spatial correlation volumes [22], which depends on the competition between the pinning strength and elasticity of the static vortex matter. If the average size of the correlation volume in the $\mathrm{ZFC}$ and FC vortex states are similar, then it is natural to expect that the $I_{c}$ and the $I-V$ curves for these states would be roughly similar. However, we find that although the $I-V$ curves are similar, the $N(B)$ curve is sensitive to differences in the ZFC and FC flowing states. This suggests that some subtle differences in the flowing vortex configuration exist deep in the FF regime. It is tempting to imagine an inhomogeneous FF state with channels of fast flowing uncorrelated vortices present alongside slowly drifting islands with relatively higher intervortex spatial correlations. A routine $I-V$ measurement is sensitive only to average $u$. However, inhomogeneity features affect $N(B)$ determined from $I-V$ cycling measurement. We argue that in the WP regime, the vortex configuration in the FF state created from the ZFC state is dominated by the slowly drifting islands. As this flow regime has significant regions with $\langle\delta\rangle \sim a_{0}$, the ZFC- $N(B)$ curve has a significant $B$ dependence (cf. Fig. 5). On the other hand, the FF regime of the FC state has more flow channels where $\langle\delta\rangle$ is not related to $a_{0}$, hence here the $N$ dependence on $B$ is weak. In fact, in the strong pinning (SP) regime, both ZFC and FC flow states are dominated by uncorrelated flow regions. Hence, in this regime as well, $N$ shows weak $B$ dependence (cf. Fig. 5). We believe that these differences in the inhomogeneity of the ZFC and FC flowing vortex states arise from subtle differences in the nature of the static vortex state prepared prior to depinning, which in turn is sensitive to the pinning regime (hence on $B$ and $T$ as well). We speculate some of these differences may arise from differences in distribution of topological defects (for example distribution of regions with fivefold to sevenfold coordinated vortices) while preparing the static vortex state. These differences may not leave a mark in the bulk $I_{c}$ values for ZFC and FC states, but they appear in the $B$ dependence of $N$. At high drives, the NDR (or quasi-NDR) transition drives the traffic of flowing vortices jammed to a halt. This jammed state has high disorder with intervortex correlation lengths at least of the order of nearest-neighbor distance. The $I_{c}$ of this state is $I_{c}{ }^{h}$, which is much higher than that achievable by any conventional means. In samples with nominal pinning, due to the ever-present intervortex interactions the static vortex state prior to depinning does not usually achieve the maximally disordered vortex configuration (intervortex correlation is at least $\sim a_{0}$ ) when prepared through any conventional means such as field cooling or accessing different pinning regime by varying $B$ and $T$. In a random pinning environment with interactions, one route to achieving a high entropy vortex configuration that is close to being maximally disordered, is via the NDR transition in the vortex flow state. In $2 \mathrm{H}-\mathrm{NbS}_{2}$, depinning from $I_{c}{ }^{h}$ is the limiting case of $I-V$ curves, as all curves with lower $I_{c}$ correspond to lower entropy vortex configurations. Considering these studies, it may be also worthwhile recalling an earlier magnetization measurement in $2 \mathrm{H}-\mathrm{NbSe}_{2}[31,53]$. It was found that minor hysteresis loop measurements initiated from the FC vortex state in these crystals with moderate pinning resulted in newer critical current density states with magnetization values lying outside the envelop magnetization hysteresis loop. The envelope loop is typically rationalized by the critical state model [54] by assuming that the critical current is a single valued function of $B$. The multivaluedness in field dependence of critical current exemplifies the higher critical current states in $2 \mathrm{H}-\mathrm{NbSe}_{2}$, which were not accessible by usual routes of field cyclings starting from $\mathrm{ZFC}$ or FC modes (e.g., see Fig. 3 of Ref. [31]). Thus, the dynamic instability-driven NDR transition in the FF state of $2 \mathrm{H}-\mathrm{NbS}_{2}$ is a route to achieve an unconventional high current density state. It is noteworthy that ac dynamics [31,55-57] produces different metastable pinned vortex configurations with different $I_{c}$ 's. These ac dynamics experiments are performed with driving ac frequencies in the range of few 10 's $\mathrm{kHz}$ and ac amplitudes of up to few 10's Oe. Compared to these ac frequencies the frequency of the repeated $I-V$ cyclings in this paper are orders of magnitude slower and the driving currents used are much higher. Here we would like to also emphasize that that the $I_{c}{ }^{h}$ state reached is not another metastable vortex pinned configuration with high $I_{c}$. The high $I_{c}{ }^{h}$ state shows distinct features such as distinct $I-V$ scaling properties around $I_{c}^{h}$, which are different from those around $I_{c}^{l}$ [25]. Also near $I_{c}^{h}$, we observe a critical slowing down of dynamics with features suggesting that the vortex state exhibits a nonequilibrium phase transition at $I_{c}{ }^{h}$ [26]. It is interesting to note that ac dynamics experiments have also shown the presence of similar slowdown of dynamics suggestive of a nonequilibrium phase transition near the plastic depinning of vortex state in MoGe thin films [58]. It is an interesting prospect for future investigations to explore the comparison of the effects of ac dynamics on the nonequilibrium driven vortex states reached via transport measurements.

\section{CONCLUSION}

In conclusion, we have shown the protocol to produce a unique disordered high $I_{c}$ state from the conventional low $I_{c}$ state. This high $I_{c}$ state is not accessible by other means. We observe unusual features related to a very high-velocity state in $2 \mathrm{H}-\mathrm{NbS}_{2}$, which we believe is related to peculiar features related to the microscopic features of the vortex core in this 
system. Our results are interpreted in terms of delocalization of bound quasiparticles inside the vortex core in $2 \mathrm{H}-\mathrm{NbS}_{2}$, which results in a drop of vortex viscosity at high drives. The consequent rapid increase in vortex velocity triggers reorganization in the flowing state leading to dynamical instability and the NDR transition. We hope that our experiments will motivate future explorations into these unusual nonequilibrium routes for reaching unusual high current density states.

\section{ACKNOWLEDGMENTS}

S.S.B. acknowledges funding support from IITK (IN) and DST-AMT-TSDP (IN), DST-SERB Imprint II (IN), Government of India. A.K.S. thanks DST for support under Year of Science Professorship. A.K.G. thanks DAE for the award of Raja Ramanna Fellowship at PEC Chandigarh. We also thank Professor A. K. Rastogi (deceased) and Professor Asad Niazi [Jamia Millia Islamia (Central University), New Delhi, 110025 , India] for the single crystals of $\mathrm{NbS}_{2}$.
[1] A. Devarakonda, H. I. Fang, C. O. Keskinbora, T. Suzuki, M. Kriener, L. Fu, E. Kaxiras, D. C. Bell, and J. G. Checkelsky, Science 370, 231 (2020).

[2] X. C. Liu, S. Zhao, X. Sun, L. Deng, X. Zou, Y. Hu, Y. X. Wang, C. W. Chu, J. Li, J. Wu, F. S. Ke, and P. M. Ajayan, Sci. Adv. 6, eaay4092 (2020).

[3] J. N. Coleman, M. Lotya, A. O'neill, S. D. Bergin, P. J. King, U. Khan, K. Young, A. Gaucher, S. De, R. J. Smith, I. V. Shvets, S. K. Arora, G. Stanton, H. Y. Kim, K. Lee, G. T. Kim, G. S. Duesberg, T. Hallam, J. J. Boland, J. J. Wang, J. F. D. Gan, J. C. Grunlan, G. Moriarty, A. Shmeliov, R. J. Nicholls, J. M. Perkins, E. M. Grieveson, K. Theuwissen, D. W. Mccomb, P. D. Nellist, and V. Nicolosi, Science 331, 568 (2011).

[4] B. V. Lotsch, Annu. Rev. Mater. Res. 45, 85 (2015).

[5] H. Ryu, Y. Chen, H. Kim, H. Z. Tsai, S. Tang, J. Jiang, F. Liou, S. Kahn, C. Jia, A. A. Omrani, J. H. Shim, Z. Hussain, Z. X. Shen, K. Kim, B. I. Min, C. Hwang, M. F. Crommie, and S. K. Mo, Nano Lett. 18, 689 (2018).

[6] B. Sipos, A. F. Kusmartseva, A. Akrap, H. Berger, L. Forro, and E. Tutis, Nature Mater. 7, 960 (2008).

[7] J. A. Wilson, F. J. DiSalvo, and S. Mahajan, Adv. Phys. 24, 117 (1975).

[8] T. Ritschel, J. Trinckauf, K. Koepernik, B. Büchner, M. v. Zimmermann, H. Berger, Y. I. Joe, P. Abbamonte, and J. Geck, Nature Phys. 11, 328 (2015).

[9] I. Gulliamon, H. Suderow, S. Vieira, L. Cario, P. Diener, and P. Rodière, Phys. Rev. Lett. 101, 166407 (2008).

[10] H. F. Hess, R. B. Robinson, and J. V. Waszczak, Phys. Rev. Lett. 64, 2711 (1990).

[11] S. Park, V. Barrena, S. M. Valero, J. J. Baldoví, A. Fente, E. Herrera, F. Mompeán, M. G. Hernández, Á. Rubio, E. Coronado, I. Guillamón, A. L.Yeyati, and H. Suderow, Nature Commun. 12, 4668 (2021).

[12] D. S. Fisher, Phys. Rep. 301, 113 (1998).

[13] G. Blatter, M. V. Feigel'man, V. B. Geshkenbein, A. I. Larkin, and V. M. Vinokur, Rev. Mod. Phys. 66, 1125 (1994).

[14] C. Reichhardt and C. J. O. Reichhardt, Rep. Prog. Phys. 80, 026501 (2017).

[15] S. Scheidl and V. M. Vinokur, Phys. Rev. E 57, 2574 (1998).

[16] A. Maeda, M. Notomi, and K. Uchinokura, Phys. Rev. B 42, 3290 (1990).

[17] F. I. B. Williams, P. A. Wright, R. G. Clark, E. Y. Andrei, G. Deville, D. C. Glattli, O. Probst, B. Etienne, C. Dorin, C. T. Foxon, and J. J. Harris, Phys. Rev. Lett. 66, 3285 (1991).

[18] C. Reichhardt, and C. J. Olson C J, N. Grønbech-Jensen, and F. Nori, Phys. Rev. Lett. 86, 4354 (2001).
[19] J. Iwasaki, M. Mochizuki, and N. Nagaosa, Nature Commun. 4, 1463 (2013).

[20] C. Reichhardt, D. Ray, and C. J. O. Reichhardt, Phys. Rev. Lett. 114, 217202 (2015).

[21] T. Giammarchi and S. Bhattacharya, Vortex Phases in High Magnetic Fields: Applications in Condensed Matter Physics and Spectroscopy edited by C Berthier, L P. Levy, and G Martinez, (Springer, Berlin, 2001), pp. 314-360 and references therein.

[22] A. I. Larkin and Y. N. Ovchinnikov, J. Low Temp. Phys. 34, 409 (1979).

[23] S. Bhattacharya and M. J. Higgins, Phys. Rev. Lett. 70, 2617 (1993).

[24] S. Mohan, J. Sinha, S. S. Banerjee, and Y. Myasoedov, Phys. Rev. Lett. 98, 027003 (2007).

[25] B. Bag, D. J. Sivananda, P. Mandal, S. S. Banerjee, A. K. Sood, and A. K. Grover, Phys. Rev. B 97, 134510 (2018).

[26] G. Shaw, P. Mandal, S. S. Banerjee, A. Niazi, A. K. Rastogi, A. K. Sood, S. Ramakrishnan, and A. K. Grover, Phys. Rev. B 85, 174517 (2012).

[27] B. Bag, G. Shaw, S. S. Banerjee, S. Majumdar, A. K. Sood, and A. K. Grover, Sci. Rep. 7, 5531 (2017).

[28] C. Reichhardt and C. J. O. Reichhardt, Phys. Rev. Lett. 103, 168301 (2009).

[29] G. Gallavotti and E. G. D. Cohen, Phys. Rev. Lett. 74, 2694 (1995).

[30] C. Baker and J. Sutton, Philos. Mag. 19, 1223 (1969); J. A. Good and E. J. Kramer, Philos. Mag. 24, 339 (1971).

[31] S. S. Banerjee, N. G. Patil, S. Ramakrishnan, and A. K. Grover, Appl. Phys. Lett. 74, 126 (1999).

[32] W. Henderson, E. Y. Andrei, M. J. Higgins, and S. Bhattacharya, Phys. Rev. Lett. 77, 2077 (1996); Z. L. Xiao, E. Y. Andrei, P. Shuk, and M. Greenblatt, ibid. 85, 3265 (2000); U. Yaron, P. L. Gammel, D. A. Huse, R. N. Kleiman, C. S. Oglesby, E. Bucher, B. Batlogg, D. J. Bishop, K. Mortensen, K. Clausen, C. A. Bolle, and F. D. L. Cruz, ibid. 73, 2748 (1994); U. Yaron, P. L. Gammel, D. A. Huse, R. N. Kleiman, C. S. Oglesby, E. Bucher, B. Batlogg, D. J. Bishop, K. Mortensen, and K. N. Clausen, Nature (London) 376, 753 (1995); S. L. Lee, G. Kealey, E. M. Forgan, S. H. Lloyd, T. M. Riseman, D. M. Paul, S. T. Johnson, C. Simon, C. Goupil, A. Pautrat, R. Cubitt, P. Schleger, C. Dewhurst, C. M. Aegerter, and C. Ager, Physica B 276-278, 752 (2000).

[33] A. D. Thakur, M. J. Higgins D.Pal, S. Ramakrishnan, and A. K.Grover, Physica C 466, 181 (2007).

[34] Y. Paltiel, E. Zeldov, Y. N. Myasoedov, H. Shtrikman, S. Bhattacharya, M. J. Higgins, Z. L. Xiao, E. Y. Andrei, 
P. L. Gammel, and D. J. Bishop, Nature (London) 403, 398 (2000).

[35] Y. Paltiel, G. Jung, Y. Myasoedov, M. L. Rappaport, E. Zeldov, M. J. Higgins, and S. Bhattacharya, Europhys. Lett. 58, 112 (2002).

[36] See Supplemental Material at http://link.aps.org/supplemental/ 10.1103/PhysRevB.104.184510 for eight sections: (I) NDR seen in a sample from a different batch section; (II) Structure and composition information; (III) $T$ dependence of $I_{c}{ }^{h}$ and $I_{c}{ }^{l}$; and demonstration of recovering back low $I_{c}{ }^{l}$ state via field cooling the sample; (IV) $I-V$ response for the jammed high $I_{c}$ state, reached in $\mathrm{R}(\mathrm{B})$ in Fig. 2 (main text); (V) All $I-V$ curves associated with Fig. 5 of the manuscript for ZFC and FC states; (VI) Nine $I-V$ cyclings. After four cycles (F1, R1, F2, R2), the high $I_{c}{ }^{h}$ state is reached (in F3). After that further cycles F3, R3, F4, R4, F5 keeps the system in the high $I_{c}{ }^{h}$ state; (VII) Temperature very near the sample during $I-V$ measurement; (VIII) Variation of dissipation with drive.

[37] A. Niazi, and A. K. Rastogi, J. Phys.: Condens. Matter 13, 6787 (2001); A. Niazi, Ph.D. thesis, School of Physical Sciences, Jawaharlal Nehru University, New Delhi, India, 1999.

[38] A. Majumdar, D. VanGennep, J. Brisbois, D. Chareev, A. V. Sadakov, A. S. Usoltsev, M. Mito, A. V. Silhanek, T. Sarkar, A. Hassan, O. Karis, R. Ahuja, and M. A. Hafie, Phys. Rev. Materials 4, 084005 (2020).

[39] J. Bardeen, and M. J. Stephen, Phys. Rev. 140, A1197 (1965); M. Tinkham, Phys. Rev. Lett. 13, 804 (1964); J. R. Clem, ibid. 20, 735 (1968).

[40] M. N. Kunchur, B. I. Ivlev, and J. M. Knight, Bull. Am. Phys. Soc. 46, 517 (2001).

[41] M. N. Kunchur, B. I. Ivlev, and J. M. Knight, Phys. Rev. Lett. 87, 177001 (2001).
[42] M. Liang and M. N. Kunchur, Phys. Rev. B 82, 144517 (2010).

[43] M. N. Kunchur, B. I. Ivlev, D. K. Christen, and J. M. Phillips, Phys. Rev. Lett. 84, 5204 (2000).

[44] R. G. Mints and A. L. Rakhmanov, Rev. Mod. Phys. 53, 551 (1981).

[45] C. Reichhardt, C. J. O. Olson, and F. Nori, Phys. Rev. B 58, 6534 (1998).

[46] V. R. Misko, S. Savel'ev, A. L. Rakhmanov, and F. Nori, Phys. Rev. Lett. 96, 127004 (2006).

[47] V. R. Misko, S. Savel'ev, A. L. Rakhmanov, and F. Nori, Phys. Rev. B 75, 024509 (2007).

[48] A. I. Larkin and Yu. N. Ovchinnikov, in Nonequilibrium Superconductivity, edited by D. N. Langenberg and A. I. Larkin, (Elsevier, Amsterdam, 1986), Chap. 11.

[49] A. Abrikosov, Fundamentals of the Theory of Metals (NorthHolland, Amsterdam, 1988).

[50] Z. E. Youbi, S. W. Jung, C. Richter, K. Hricovini, C. Cacho, and M. D. Watson, Phys. Rev. B 103, 155105 (2021).

[51] C. Witteveen, K. Górnicka, J. Chang, M. Månsson, T. Klimczuk, and F. O. von Rohr, Dalton Trans. 50, 3216 (2021).

[52] S. Mohan, J. Sinha, S. S. Banerjee, A. K. Sood, S. Ramakrishnan, and A. K. Grover, Phys. Rev. Lett. 103, 167001 (2009).

[53] S. S. Banerjee, Ph.D. thesis, University of Mumbai, India, 2000.

[54] C. P. Bean, Phys. Rev. Lett. 8, 250 (1962).

[55] S. O. Valenzuela and V. Bekeris, Phys. Rev. Lett. 84, 4200 (2000).

[56] S. O. Valenzuela and V. Bekeris, Phys. Rev. Lett. 86, 504 (2001).

[57] G. Pasquini, M. Marziali Bermúdez, and V. Bekeris, Supercond. Sci. and Technol. 34, 013003 (2020).

[58] S. Okuma, Y. Tsugawa, and A. Motohashi, Phys. Rev. B 83, 012503 (2011). 